\title{
ON DEVIATIONS FROM LINEAR WAVE MOTION IN INHOMOGENEOUS STARS*
}

\author{
$\mathrm{By}$ \\ P. J. MELVIN \\ University of Illinois at Urbana-Champaign
}

\begin{abstract}
The hydrodynamic equations for the large-amplitude, adiabatic pulsations of a spherically symmetric, inhomogeneous star are solved by a method of approximation in which the form of the fluid velocity is specified a priori. The assumed velocity is a nonlinear function of the radius and contains two arbitrary functions of time. These two functions are determined by a pair of second-order, quasi-linear, ordinary differential equations, and an analytic, periodic solution to these equations is constructed. This solution corresponds to large amplitude, anharmonic, nonlinear pulsations of a star in which the fluid velocity is a travelling wave. A specific inhomogeneous star is studied to demonstrate the feasibility of numerically solving the pair of differential equations and of constructing the periodic solution.
\end{abstract}

1. Introduction. It is shown by Rosseland [1] that the hydrodynamic equations for the adiabatic motion of an homogeneous, spherically symmetric star admit an analytical solution when the fluid velocity is a separable function of radius and time. This Eulerian velocity has been called a linear wave velocity by McVittie [2], Disney, McNally and Wright [3] among others because its positional dependence is proportional to the radial coordinate. Lin, Mestal and Shu [4], Fujimoto [5] and others have investigated the motions of ellipsoidal, self-gravitating, homogeneous distributions of matter. In these cases the density is assumed to depend only on time, and the Eulerian velocity is assumed to depend linearly on position.

In this paper the adiabatic motions of non-radiating, spherically symmetric stars are investigated for cases in which the Eulerican velocity is assumed to be a nonlinear function of position and the density is assumed to depend both on the radial coordinate and time. In Sec. 2 the nonlinear velocity is introduced; a Lagrangian radial coordinate is found; the density and pressure are found in terms of arbitrary functions; and the condition of motion about hydrostatic equilibrium is imposed.

The assumed velocity law involves two functions of time and a theory is developed in Sec. 3 to determine these functions. It is not possible to solve for the adiabatic motion of an inhomogeneous star in a pointwise exact manner. Two ordinary differential equations for the two functions of time are derived, however, from the condition that the solution to the hydrodynamic equations is "globally" exact.

* Received April 16, 1976; revised version received September 8, 1976. The author wishes to acknowledge the helpful advice of Prof. G. C. McVittie in preparing this article, and the programming assistance of Mr. J. M. Michl. 
In Sec. 4 the two nonlinear differential equations are transformed into a "quasilinear" form, and in Sec. 5 various special and approximate solutions are found. Two other possible equilibrium solutions are obtained and the known solution for an homogeneous star is reviewed in the present notation. The method of variation of parameters of an approximate solution is used to obtain an alternative set of three first order differential equations. Next a single parameter family of large-amplitude, anharmonic, periodic solutions for an inhomogeneous star is obtained by expansion of the solution in terms of a certain constant $\delta$. This constant vanishes for an homogeneous star, and the solution is constructed so that it reduces to the known solution for an homogeneous star in the limit as $\delta$ tends to zero. A simple inhomogeneous star is treated in Sec. 6 and curves of constant energy are given. The nature of the additional equilibrium points is discussed, and the results of two numerical integrations of the first-order equations are presented. Five members of the family of periodic solutions are given by graphs. In the final section the possible observable quantities of this theory are obtained.

\section{Kinematics of the assumed velocity law.}

2.1 A nonlinear velocity law. The hydrodynamical motion of a spherically symmetric star can be described in terms of an Eulerian velocity $v(r, t)$. The only boundary condition on this velocity is that it vanishes at the center of the star for all times; that is, $v(r=0, t)=0$. It is assumed that the amplitude of the motion of the star may be large, but also that it is sufficiently "smooth" that the radial dependence of $v$ can be developed in a Taylor series about $r=0$. Only the first two terms of this series are retained, and it is assumed that these two terms involve the first and third powers of $r$. The coefficients of these two terms depend only on time, and for later simplicity the velocity law is written as

$$
v(r, t)=r\left[\dot{R}(t) / R(t)-\frac{1}{2} \dot{a}(t)(r / R(t))^{2}\right] .
$$

The "undetermined coefficients" $R(t)$ and $a(t)$ depend only on time, and dots are used to denote time differentiation. When $\dot{a}(t)$ is identically zero, the velocity law (2.101) reduces to the well-known linear wave velocity. To the best of the author's knowledge, the consequences of the nonlinear velocity law (2.101) have not been studied before.

2.2 A Lagrangian radial coordinate. In spherical symmetry it is often convenient to proceed in terms of the Lagrangian scheme of hydrodynamics. The connection between the Eulerian and Lagrangian formulations is found by integration of the ordinary differential equation

$$
\dot{r}(t)=v(r, t) .
$$

The constant of integration obtained by integration of this equation is the Lagrangian or co-moving radial coordinate of the gas shells. When (2.101) is substituted in (2.201) Bernoulli's equation results, and it is integrated as

$$
r(\xi, t)=R(t) \xi\left[1+a(t) \xi^{2}\right]^{-1 / 2},
$$

where $\xi$ is a dimensionless constant of integration. The form (2.202) is chosen in order that linear wave results should hold when $a(t) \equiv 0$. The constant of integration $\xi$ is interpreted as a label for the shells of gas which make up the star; hence, the radial coordinate $r$ of the $\xi$ th shell of gas is computed from (2.202) at all times when $R(t)$ and $a(t)$ are given. Only finite distributions of gas are considered, and it is assumed that 
they have an outer boundary at $\xi=1$. Thus, the radius of the boundary $r_{b}(t)$ is computed from (2.202) as

$$
r_{o}(t)=R(t)[1+a(t)]^{-1 / 2}
$$

It is assumed that the star oscillates about some equilibrium configuration which is defined to occur when $R=R_{0}$, a constant, and $a=0$. From (2.203), $R_{0}$ can be interpreted as the equilibrium radius of the star.

2.3 The density distribution. In the Lagrangian scheme, the radial and temporal dependence of the density $\rho(\xi, t)$ is easily determined by differentiation of $r(\xi, t)$. The Lagrangian continuity equation is

$$
\rho(\xi, t)=\rho_{0}(\xi) /\left[(r / \xi)^{2}(\partial r / \partial \xi)\right],
$$

where $\rho_{0}(\xi)$ is some non-negative function which vanishes for $\xi>1$. From (2.202) the density (2.301) is obtained as

$$
\rho(\xi, t)=\left[1+a(t) \xi^{2}\right]^{5 / 2} \rho_{0}(\xi) / R^{3}(t) .
$$

In equilibrium, $R=R_{0}, a=0$, and the function $\rho_{0}(\xi)$ is related to the equilibrium density $\rho_{e}(\xi)$ by $(2.302)$ as

$$
\rho_{0}(\xi)=R_{0}{ }^{3} \rho_{e}(\xi), \quad \xi=r / R_{0} .
$$

2.4 The pressure distribution for adiabatic motion. If only adiabatic transformations are considered, the "adiabatic pressure" $P_{a}$ is obtained from

$$
P_{a}(\xi, t)=\left[\rho(\xi, t) / \rho_{0}(\xi)\right]^{5 / 3} P_{0}(\xi),
$$

where $P_{0}(\xi)$ is some non-negative function which vanishes for $\xi \geq 1$ and it is assumed that the ratio of specific heats is $5 / 3$. From (2.302) and (2.401) there is obtained

$$
P_{a}(\xi, t)=\left[1+a(t) \xi^{2}\right]^{25 / 8} P_{0}(\xi) / R^{5}(t) .
$$

In equilibrium, $R=R_{0}, a=0$, and the adiabatic pressure is assumed to reduce to the equilibrium pressure $P_{e}(\xi)$. By (2.402) these functions are related by

$$
P_{0}(\xi)=R_{0}{ }^{5} P_{e}(\xi), \quad \xi=r / R_{0} .
$$

The equilibrium density and pressure distributions are related by the equation of hydrostatic equilibrium

$$
d P_{e}(r) / d r=-G \rho_{e}(r) m(r) / r^{2},
$$

where $G$ is the constant of gravitation and $m(r)$ is the mass interior to the radius $r$. In terms of $\rho_{0}(\xi)$ and $P_{0}(\xi)$, the last equation is by (2.303) and (2.403)

$$
d P_{0}(\xi) / d \xi=-G R_{0} \rho_{0}(\xi) m(\xi) / \xi^{2}
$$

where

$$
m(\xi)=4 \pi \int_{0}^{\xi} \eta^{2} \rho_{0}(\eta) d \eta
$$

The techniques for integration of (2.404) can be found in books on stellar structure; see, for example, Cox and Giuli [6]. The boundary condition on the ordinary differential equation (2.404) is that $P_{0}(\xi=1)=0$. The complications due to radiative transfer 
and nuclear energy generation are too difficult to be considered here. As a result there is only one equation (2.404) between the unknown functions $P_{0}(\xi)$ and $\rho_{0}(\xi)$. Throughout what follows it is assumed that $\rho_{0}(\xi)$ is some given positive function of $\xi$, and that $P_{0}(\xi)$ is computed by integration of (2.404).

\section{Dynamical considerations.}

3.1 The method of approximation. The force equation for a star can be written in the Lagrangian scheme as

$$
\partial P_{m} / \partial \xi=-\rho(\partial r / \partial \xi)\left(\partial^{2} r / \partial t^{2}+G m / r^{2}\right)
$$

where $P_{m}(\xi, t)$ is called the "momentum" pressure to distinguish it from the "adiabatic" pressure $P_{a}(\xi, t)$ which is computed from (2.402). If $\rho_{0}(\xi), R(t)$ and $a(t)$ are given, then the right-hand side of (3.101) is determined. Thus, $P_{m}(\xi, t)$ can be obtained by integration of (3.101) with the boundary condition that $P_{m}(\xi=1, t)=0$.

In general, if $\rho_{0}(\xi), R(t)$ and $a(t)$ are given arbitrarily, the pressures computed from (2.402) and (3.101) will not agree. The difference in the pressures $\epsilon(\xi, t)$ is defined by

$$
\epsilon(\xi, t)=P_{m}(\xi, t)-P_{a}(\xi, t) .
$$

This difference vanishes identically in two cases: 1 ) in equilibrium when $R \equiv R_{0}, a \equiv 0$ and $\rho_{0}(\xi)$ arbitrary; 2$)$ for a homogeneous star for which $\rho_{0}(\xi)$ is a constant, $R$ is a computable function of $t$ and $a \equiv 0$. Although these are the only two cases where it can be guaranteed that $\epsilon(\xi, t) \equiv 0$, there should be some general way of proceeding so that $|\epsilon(\xi, t)|$ is tolerably small when $R, a$ and $\rho_{0}$ are not constant. The precedure which is followed here is to choose $R(t)$ and $a(t)$, leaving $\rho_{0}(\xi)$ arbitrary, such that the integral of $\epsilon$ over the volume of the star always vanishes; that is,

$$
\int_{0}^{1} r^{2} \epsilon(\partial r / \partial \xi) d \xi=0
$$

For $R(t)$ and $a(t)$ chosen in this way, the resulting solution to the hydrodynamic equations for the adiabatic motion of a star is "globally exact" in the sense of (3.103), but since in general $\epsilon(\xi, t) \neq 0$, it is pointwise in error.

If the initial restrictive assumption (2.101) about the nature of the gas flow were not made, there would be no distinction between the adiabatic and momentum pressures, the total energy of the star would be derivable from the force euqation, and the total energy would be constant for all possible adiabatic motions of a star. The procedure used here to approximate some of these motions with the assumption (2.101) is as follows: the total energy of the star is computed from the adiabatic pressure, the resulting energy is treated as the Hamiltonian for a mechanical system with two degrees of freedom, and two second-order, ordinary differential equations are derived from Hamilton's equations. Any solution to this pair of equations then yields an $R(t)$ and $a(t)$ such that (3.103) holds.

3.2 The total kinetic energy. The total kinetic energy (K.E.) of the star is the integral over the volume of the star of the kinetic energy per unit volume, $\frac{1}{2} \rho v^{2}$. In the Lagrangian scheme this integral is

$$
\text { K.E. }=2 \pi \int_{0}^{1} \xi^{2} \rho_{0}(\xi)[\partial r(\xi, t) / \partial t]^{2} d \xi .
$$

When (2.202) is substituted in this expression, there is obtained 


$$
\text { K.E. }=2 \pi \rho_{c}\left[\dot{R}^{2} u(a)+\dot{R} R \dot{a} u^{\prime}(a)+\frac{1}{8} R^{2} \dot{a}^{2} u^{\prime \prime}(a)\right],
$$

where the constant $\rho_{c}$ and the function $u(a)$ are defined by

$$
\rho_{c}=\int_{0}^{1} \xi^{4} \rho_{0}(\xi) d \xi, \quad \rho_{c} u(a)=\int_{0}^{1} \xi^{4} \rho_{0}(\xi)\left(1+a \xi^{2}\right)^{-1} d \xi,
$$

and where, here and below, primes denote differentiation with respect to the argument indicated.

Two transformations reduce (3.201) to a simple diagonal form. If $q(R, a)$ is defined by

$$
q=[u(a)]^{1 / 2} R
$$

then (3.201) becomes

$$
\text { K.E. }=2 \pi \rho_{c}\left[\dot{q}^{2}+q^{2} \dot{a}^{2} w(a) / 8 u^{2}(a)\right],
$$

where $w(a)$ is defined by

$$
w(a)=u(a) u^{\prime \prime}(a)-2\left[u^{\prime}(a)\right]^{2} .
$$

If $\rho_{0}(\xi)$ is a positive integrable function, it can be shown that $u(a)$ and $w(a)$ are positive, finite functions for $-1<a<\infty$. Thus, the function $\theta(a)$ defined by

$$
d \theta / d a=(w / 2)^{1 / 2} / 2 u, \quad \theta(a=0)=0,
$$

is a well-defined, invertible function of $a$. With this second transformation (3.204) becomes

$$
\text { K.E. }=2 \pi \rho_{c}\left(\dot{q}^{2}+q^{2} \dot{\theta}^{2}\right) .
$$

3.3 The internal energy. The total internal energy (I.E.) of the star is the integral over the volume of the star of the internal energy per unit volume, $\frac{3}{2} P$; that is,

$$
\text { I.E. }=6 \pi \int_{0}^{1} r^{2} P(\partial r / \partial \xi) d \xi .
$$

It is assumed that the total internal energy of the star is identical for both $P_{a}$ and $P_{m}$ so that (3.103) holds. By (2.202) and (2.402) the last equation becomes

$$
\text { I.E. }=6 \pi R^{-2} \int_{0}^{1} \xi^{2}\left(1+a \xi^{2}\right)^{5 / 3} P_{0}(\xi) d \xi .
$$

The transformation (3.203) in this equation yields

$$
\text { I.E. }=6 \pi P_{c} V_{1}(\theta) / q^{2},
$$

where the constant $P_{c}$ and the "potential" $V_{1}(\theta)$ are defined by

$$
P_{c}=\int_{0}^{1} \xi^{2} P_{0}(\xi) d \xi, \quad P_{c} V_{1}(\theta)=u(a) \int_{0}^{1} \xi^{2}\left(1+a \xi^{2}\right)^{5 / 3} P_{0}(\xi) d \xi,
$$

and where $a=a(\theta)$ is the inverse of the transformation (3.206).

3.4 The gravitational potential energy. The total gravitational potential energy $(\Omega)$ of the star is the integral over the volume of the star of the gravitational potential energy per unit volume, $-G \rho m / r$. In spherical symmetry this is

$$
\Omega=-4 \pi G \int_{0}^{1} r^{-1} \xi^{2} \rho_{0}(\xi) m(\xi) d \xi
$$


From (2.202) the last expression becomes

$$
\Omega=-4 \pi G R^{-1} \int_{0}^{1} \xi\left(1+a \xi^{2}\right)^{1 / 2} \rho_{0}(\xi) m(\xi) d \xi .
$$

The transformation (3.203) then yields

$$
\Omega=-4 \pi G \rho_{c} M K V_{2}(\theta) / q,
$$

where the total mass of the star $M$ and the "structure constant" $K$ are defined by

$$
M=4 \pi \int_{0}^{1} \xi^{2} \rho_{0}(\xi) d \xi, \quad K=\int_{0}^{1} \xi \rho_{0}(\xi) m(\xi) d \xi / \rho_{c} M,
$$

where the "potential" $V_{2}(\theta)$ is computed from

$$
\rho_{c} M K V_{2}(\theta)=u^{1 / 2}(a) \int_{0}^{1} \xi\left(1+a \xi^{2}\right)^{1 / 2} \rho_{0}(\xi) m(\xi) d \xi
$$

and where $a=a(\theta)$ is computed as the inverse to (3.206). From (3.202), (3.302) and (3.403) the functions $u(a), V_{1}(\theta)$ and $V_{2}(\theta)$ are dimensionless and have the value unity at $a=\theta=0$.

A connection between $P_{c}(3.302)$ and $K(3.402)$ is obtained from (3.302) by an integration by parts and (2.404) as

$$
P_{c}=\frac{1}{3} G \rho_{c} M R_{0} K
$$

From this, (3.301) becomes

$$
\text { I.E. }=2 \pi G \rho_{c} M R_{0} K V_{1}(\theta) / q^{2} .
$$

3.5 The total energy. The constant total energy of the star is denoted by $4 \pi \rho_{c} E_{0}$ and is the sum of the total kinetic, internal and gravitational potential energies. Thus, from (3.207), (3.405) and (3.401) there is obtained

$$
E_{0}=\frac{1}{2}\left(\dot{q}^{2}+q^{2} \dot{\theta}^{2}\right)+G M K\left(\frac{1}{2} R_{0} V_{1}(\theta) / q^{2}-V_{2}(\theta) / q\right) .
$$

If (3.501) is considered to be the Hamiltonian for a dynamical system with two degrees of freedom represented by $q$ and $\theta$, then Hamilton's equations (see for example Goldstein [7]) yield

$$
\begin{aligned}
\ddot{q}-q \dot{\theta}^{2} & =G M K\left[R_{0} V_{1}(\theta) / q^{3}-V_{2}(\theta) / q^{2}\right], \\
d\left(q^{2} \dot{\theta}\right) / d t & =G M K\left[-\frac{1}{2} R_{0} V_{1}^{\prime}(\theta) / q^{2}+V_{2}^{\prime}(\theta) / q\right] .
\end{aligned}
$$

These two differential equations have the integral (3.501).

In summary, it is assumed that $\rho_{0}(\xi)$ and $R_{0}$ are given. The function $P_{0}(\xi)$ is computed for this $\rho_{0}(\xi)$ from the equation of hydrostatic equilibrium (2.404). The constants $\rho_{c}, P_{c}$, $M$ and $K$ are computed from (3.202), (3.302) or (3.404) and (3.402), respectively. The function $u(a)$ and its derivatives are computed from the second equation of (3.202). The function $\theta(a)$ and its inverse $a(\theta)$ are computed from (3.206). The potentials $V_{1}(\theta)$ and $V_{2}(\theta)$ are computed from (3.302) and (3.403). With $V_{1}(\theta)$ and $V_{2}(\theta)$ determined, (3.502) and (3.503) may be integrated to yield $q(t)$ and $\theta(t)$ which guarantee that (3.103) holds. Then $R(t)$ and $a(t)$ are found from $q(t)$ and $\theta(t)$ by the inverses to the transformations (3.203) and (3.206). Hence, $r(\xi, t), \rho(\xi, t)$ and $P_{a}(\xi, t)$ can be computed for this $R(t)$ and $a(t)$ by (2.202), (2.302) and (2.402). 


\section{A quasi-linear set of equations.}

4.1 A general transformation. The equations (3.502) and (3.503) exhibit a certain simple structure in the variable $q$ which is analogous to the form of the equations in the Newtonian two-body problem (see, for example, Danby [8]). To take advantage of this, the dependent variable $Z(\varphi)$ and the independent variable $\varphi$ are defined as

$$
Z=R_{0} / q, \quad d \varphi / d t=Z^{2} / T_{0},
$$

where $T_{0}$ is a constant with the units of time; hence, $Z$ and $\varphi$ are dimensionless. With these transformations (3.501), (3.502) and (3.503) become

$$
\begin{gathered}
\frac{1}{2}\left[Z^{\prime 2}(\varphi)+\left(\theta^{\prime 2}(\varphi)+V_{1}(\theta)\right) Z^{2}\right]-V_{2}(\theta) Z=E, \\
Z^{\prime \prime}(\varphi)+\left({\theta^{\prime}}^{2}(\varphi)+V_{1}(\theta)\right) Z=V_{2}(\theta), \\
\theta^{\prime \prime}(\varphi)+\frac{1}{2} V_{1}^{\prime}(\theta)=V_{2}^{\prime}(\theta) / Z,
\end{gathered}
$$

where $E=E_{0}\left(T_{0} / R_{0}\right)^{2}$ is dimensionless and where $T_{0}$ is chosen to satisfy

$$
G M K T_{0}{ }^{2} / R_{0}{ }^{3}=1 .
$$

The advantage of the transformations (4.101) is that the left-hand side of (4.103) is linear in $Z$; the disadvantage is that (4.101) requires an additional integration to obtain $t(\varphi)$.

4.2 Small amplitude in $\theta$. It is now assumed that $|\theta(t)|$ is small and, therefore, that $V_{1}(\theta)$ and $V_{2}(\theta)$ can be expanded in a Taylor series and truncated at the quadratic power of $\theta$, namely,

$$
\begin{aligned}
& V_{1}(\theta)=1+2 \delta \theta+\alpha \theta^{2}, \\
& V_{2}(\theta)=1+\delta \theta+\frac{1}{2} \beta \theta^{2} .
\end{aligned}
$$

It should be recalled that $u, V_{1}$ and $V_{2}$ are unity at $a=\theta=0$. The coefficients of $\theta$ in these two expressions are $2 \delta$ and $\delta$ because (2.404) is assumed to hold. Recourse to the definitions (3.302) and (3.403) along with the use of the chain rule, (2.404) and (3.206) yields eventually for the constants in (4.201) and (4.202)

$$
\begin{gathered}
\delta=[2 / w(0)]^{1 / 2}\left[u^{\prime}(0)+\int_{0}^{1} \xi^{3} \rho_{0} m d \xi / \int_{0}^{1} \xi \rho_{0} m d \xi\right], \\
\alpha=[4 / w(0)]\left[u^{\prime \prime}(0)+\left(2 u^{\prime}(0) \int_{0}^{1} \xi^{3} \rho_{0} m d \xi+\frac{10}{21} \int_{0}^{1} \xi^{5} \rho_{0} m d \xi\right) / \int_{0}^{1} \xi \rho_{0} m d \xi\right] \\
+[2 / w(0)]^{1 / 2} \delta\left[u^{\prime}(0)-w^{\prime}(0) / 2 w(0)\right], \\
\beta=[4 / w(0)]\left[-\frac{1}{2} u^{\prime 2}(0)+u^{\prime \prime}(0)+\left(u^{\prime}(0) \int_{0}^{1} \xi^{3} \rho_{0} m d \xi\right.\right. \\
\left.\left.-\frac{1}{2} \int_{0}^{1} \xi^{5} \rho_{0} m d \xi\right) / \int_{0}^{1} \xi \rho_{0} m d \xi\right]+[2 / w(0)]^{1 / 2} \delta\left(2 u^{\prime}-w^{\prime} / w\right)
\end{gathered}
$$

where $w(0), u^{\prime}(0)$, etc. denote the values of the functions $w(a), u^{\prime}(a)$, etc. at $a=0$. These dimensionless constants involve only integrals of $\rho_{0}(\xi)$ and may be computed once $\rho_{0}(\xi)$ is specified.

When (4.201) and (4.202) are substituted in (4.103) and (4.104) there is obtained 


$$
\begin{aligned}
Z^{\prime \prime}+\left(1+{\theta^{\prime}}^{2}+2 \delta \theta+\alpha \theta^{2}\right) Z & =1+\delta \theta+\frac{1}{2} \beta \theta^{2}, \\
Z \theta^{\prime \prime}+(\alpha Z-\beta) \theta & =\delta(1-Z) .
\end{aligned}
$$

The dependence of the left-hand sides of (4.206) and (4.207) is linear in $Z$ and $\theta$, respectively, but the coupling is nonlinear, so it is appropriate to call (4.206) and (4.207) quasi-linear.

5. Approximate and special solutions. From the summary at the end of Sec. 3.5, the essential problem of the theory developed in this paper is the solution of the equations (3.502) and (3.503). Even though these equations have been reduced to the quasi-linear set (4.206) and (4.207) which are correct to order $\theta^{2}$, it is by no means a simple task to integrate these equations exactly. By investigating various numerical solutions to these equations, this author has discovered various special and approximate solutions which are given next.

5.1 Equilibrium solutions. An "equilibrium solution" to (4.206) and (4.207) is defined to be any solution for which $Z$ and $\theta$ are constants. Since the theory of this paper has assumed that the star oscillates about a state of hydrostatic equilibrium, it is to be expected that (4.206) and (4.207) should have at least one equilibrium solution. This expected solution is $Z=1, \theta=0$. However, this is not the only equilibrium solution. If $Z^{\prime \prime}$ and $\theta^{\prime}$ are set equal to zero in (4.206), there is obtained

$$
Z=\left(1+\delta \theta+\frac{1}{2} \beta \theta^{2}\right) /\left(1+2 \delta \theta+\alpha \theta^{2}\right) .
$$

If $\theta^{\prime \prime}$ is set equal to zero in (4.207) and if (5.101) is used to eliminate $Z$, there is obtained $\theta\left[\alpha \beta \theta^{2}+3 \delta \beta \theta-2\left(\alpha-\beta-\delta^{2}\right)\right]=0$.

There are three values of $\theta$ which satisfy this cubic equation, namely, $\theta=0$ and

$$
\theta=-3 \delta / 2 \alpha \pm\left[2(\alpha-\beta) / \alpha \beta+\delta^{2}(9 \beta-8 \alpha) / 4 \alpha^{2} \beta\right]^{1 / 2} .
$$

Each of these values of $\theta$ has a corresponding value of $Z$ which is computed from (5.101). Thus, as long as the radical in (5.102) is real, (4.206) and (4.207) have three equilibrium solutions.

5.2 The homogeneous star. To illustrate the foregoing theory, and to summarize the known results in the present notation, the case of a homogeneous star is considered in this section. It is assumed that

$$
\rho_{0}(\xi)=3 M / 4 \pi
$$

where $M$ is the constant total mass of the star. Following the summary at the end of Sec. 3.5, it is found from (2.405) and (2.404) that

$$
m(\xi)=M \xi^{3}, \quad P_{0}(\xi)=3 G M^{2} R_{0}\left(1-\xi^{2}\right) / 8 \pi .
$$

From (3.202), (3.302) and (3.402) there comes

$$
\rho_{c}=3 M / 20 \pi, \quad P_{c}=G M^{2} R_{0} / 20 \pi, \quad K=1 .
$$

The function $u(a)$ can be obtained in terms of elementary functions from the definition (3.202) as

$$
u(a)=5\left[\frac{1}{3} a+\left(u_{0}(a)-1\right) / a^{2}\right]
$$

where $u_{0}(a)$ is defined as 


$$
\begin{array}{rlrl}
u_{0}(a)=\int_{0}^{1}\left(1+a \xi^{2}\right)^{-1} d \xi & \\
& =a^{-1 / 2} \arctan a^{1 / 2}, & & a>0 \\
& =1 & & a=0 \\
& =\frac{1}{2}(-a)^{1 / 2} \ln \left\{\left[1+(-a)^{1 / 2}\right] /\left[1-(-a)^{1 / 2}\right]\right\}, & & a<0 .
\end{array}
$$

The formula (5.204) is not useful for numerical evaluation near $a=0$; hence, a power series for $u(a)$ which converges for $|a|<1$ is derived from (3.202) as

$$
u(a)=5 \sum_{n=0}^{\infty}(-1)^{n} a^{n} /(2 n+5) .
$$

From the last formula $u^{\prime}(0), u^{\prime \prime}(0)$ and $u^{\prime \prime \prime}(0)$ may be easily computed.

The relation between $a$ and $\theta$ is found to order $\theta^{2}$ from (3.206) as

$$
a(\theta)=2[2 / w(0)]^{1 / 2} \theta+[2 / w(0)]\left[2 u^{\prime}(0)-w^{\prime}(0) / w(0)\right] \theta^{2} .
$$

When (5.206) is used, the numerical coefficients in (5.207) are found as

$$
a(\theta)=9.39153 \theta+278.817 \theta^{2} .
$$

It has been assumed that the potentials $V_{1}(\theta)$ and $V_{2}(\theta)$ are adequately approximated by (4.201) and (4.202). The constants $\alpha, \beta$ and $\delta$ are computed from (4.203), (4.204) and (4.205) for the density (5.201) as

$$
\delta=0, \quad \alpha=47 / 3, \quad \beta=3 .
$$

For the homogeneous star the equilibrium solutions (5.102) and (5.101) are obtained from (5.209) as

$$
Z=1, \quad \theta=0 ; \quad Z=0.191489, \quad \theta= \pm 0.734171 .
$$

With the constants (5.209), Eqs. (4.206) and (4.207) reduce to

$$
\begin{aligned}
Z^{\prime \prime}+\left(1+\theta^{\prime 2}+\alpha \theta^{2}\right) Z & =1+\frac{1}{2} \beta \theta^{2}, \\
\theta^{\prime \prime}+(\alpha-\beta / Z) \theta & =0 .
\end{aligned}
$$

This pair of equations has the particular solution

$$
Z(\varphi)=1+e \cos \varphi, \quad \theta(\varphi) \equiv 0,
$$

where $e$ is a constant of integration called the "skewness parameter." In the sequel the solution (5.213) is referred to as the "homogeneous solution." In the present notation, the homogeneous solution is the time part of the separable velocity which Rosseland [1] obtained in his Sec. 7.4 for an homogeneous star.

For the homogeneous solution, the connection between $t$ and $\varphi$ can be found in terms of elementary functions. When (5.213) is substituted in the second equation of (4.101), the integral which results is most easily performed by tangents of half angles. Thus, when $0 \leq e<1$ the parameter $\mu$ is defined as

$$
\tan \frac{1}{2} \mu=[(1-e) /(1+e)]^{1 / 2} \tan \frac{1}{2} \varphi,
$$

and the integral is found to be

$$
\left(t-t_{0}\right) / T=(2 \pi)^{-1}(\mu-e \sin \mu)
$$


where $t_{0}$ is a constant of integration and $T$ is the time period of the oscillation given by

$$
T=2 \pi\left(1-e^{2}\right)^{-3 / 2} T_{0} .
$$

From (4.105) and (5.203) the period is

$$
T=2 \pi(G M)^{-1 / 2}\left[R_{0} /\left(1-e^{2}\right)\right]^{3 / 2},
$$

which relates the time period to the mass $M$, radius $R_{0}$ and amplitude of the motion $e$.

It should be noted that even if the quadratic terms in $\theta$ are neglected in (5.211), the general solution to the pair of equations (5.211) and (5.212) is not a simple task. For in this case (5.212) becomes with the first equation of (5.213)

$$
\theta^{\prime \prime}+[\alpha-\beta /(1+e \cos \varphi)] \theta=0 .
$$

The coefficients of this linear, second-order differential equation are periodic, and for $|e|<1,(5.215)$ is Hill's differential equation (Hill [9]). The solution to such an equation may or may not be a bounded function, and an investigation of the stability of (5.215) is beyond the scope of this paper.

5.3 Approximate general solution. It is obvious that (5.215) can be immediately integrated if $\beta=0$. In this and only this section it is assumed that $\beta=\delta=0$ and the general solution to (4.206) and (4.207) is constructed. With this assumption (4.207) is independent of $Z$; hence, the coefficient of $Z$ in (4.206) must be a constant of integration. If this constant is denoted by $S^{2}$, there is obtained from the energy integral (4.102) with (4.201) and (4.202)

$$
\frac{1}{2}\left[Z^{\prime 2}+S^{2} Z^{2}\right]-Z=E, \quad \theta^{\prime 2}+1+\alpha \theta^{2}=S^{2} .
$$

This pair of equations may be integrated as

$Z=S^{-2}\left[1+\left(1+2 S^{2} E\right)^{1 / 2} \cos S \varphi\right], \quad \theta=\left[\left(S^{2}-1\right) / \alpha\right]^{1 / 2} \cos \sqrt{\alpha}\left(\varphi-\varphi_{0}\right)$,

where $E, S$ and $\varphi_{0}$ are constants of integration. The fourth constant of integration amounts to a trivial shift of the solution (5.302) along the $\varphi$-axis and is suppressed. The general solution to (4.206) and (4.207) when $\beta=\delta=0$ is written in the form (5.302) for comparison with later results.

5.4 First-order equations. The general solution to the pair of second-order equations (4.206) and (4.207) must involve four constants of integration. One constant is known by the energy integral (4.102). In this section an alternative set of three first-order equations is derived which explicitly contain the energy constant.

By analogy with the preceeding section, the function $S(\varphi)$ is defined by

$$
S^{2}(\varphi)={\theta^{\prime}}^{2}+V_{1}(\theta)=1+{\theta^{\prime}}^{2}+2 \delta \theta+\alpha \theta^{2} .
$$

With this definition (4.102) and (4.206) become

$$
\begin{gathered}
Z^{\prime 2}+S^{2} Z^{2}-2 V_{2} Z=2 E, \\
Z^{\prime \prime}+S^{2} Z=V_{2} .
\end{gathered}
$$

If (5.402) is differentiated with respect to $\varphi$ and if the equation which results is compared with (5.403), a differential equation for $S(\varphi)$ is obtained as

$$
d S^{2} / d \varphi=2 V_{2}^{\prime}(\theta) \theta^{\prime}(\varphi) / Z(\varphi)
$$

which is the first equation. 
The form of the energy integral (5.402) can be changed by completing squares to

$$
Z^{\prime 2}=S^{2}\left[\left(V_{2}^{2}+2 S^{2} E\right) / S^{4}-\left(Z-V_{2} / S^{2}\right)^{2}\right] .
$$

Since $Z^{\prime 2}$ cannot be negative, (5.405) determines the amplitude of $Z$. To take advantage of this, the function $X(\varphi)$ is defined by

$$
Z=S^{-2}\left[V_{2}+\left(V_{2}^{2}+2 S^{2} E\right)^{1 / 2} \cos X\right]
$$

The function $Z^{\prime}(\varphi)$ can be computed in two ways: by differentiation of (5.406) and by substitution of (5.406) in (5.405). If these two results are equated, a differential equation for $X(\varphi)$ is obtained. Hence, substitution of (5.406) in (5.405) gives

$$
Z^{\prime}=-S^{-1}\left(V_{2}^{2}+2 S^{2} E\right)^{1 / 2} \sin X
$$

where the negative root is chosen so that $Z^{\prime}$ is negative when $Z$ decreases. Differentiating (5.406) with respect to $\varphi$ yields eventually

$$
Z^{\prime}=-\left(V_{2} V_{2}^{\prime} \theta^{\prime} / S^{4} Z\right) \sin ^{2} X-S^{-2} X^{\prime}\left(V_{2}^{2}+2 S^{2} E\right)^{1 / 2} \sin X,
$$

where (5.404) and (5.406) have been used. When (5.407) and (5.408) are equated, a differential equation for $X$ is obtained as

$$
X^{\prime}(\varphi)=S-V_{2}(\theta) V_{2}{ }^{\prime}(\theta) \theta^{\prime}(\varphi)\left[S^{2} Z\left(V_{2}{ }^{2}+2 S^{2} E\right)^{1 / 2}\right]^{-1} \sin X,
$$

which is the second equation.

By completing squares (5.401) can be rewritten as

$$
{\theta^{\prime}}^{2}=\alpha\left[\left(\left(S^{2}-1\right) \alpha+\delta^{2}\right) / \alpha^{2}-(\theta+\delta / \alpha)^{2}\right] .
$$

Since $\theta^{\prime 2}$ cannot be negative (5.410) determines the amplitude of $\theta$. The function $Y(\varphi)$ is defined by

$$
\theta=\alpha^{-1}\left\{-\delta+\left[\left(S^{2}-1\right) \alpha+\delta^{2}\right]^{1 / 2} \cos Y\right\} .
$$

Substitution of (5.411) in (5.410) gives

$$
\theta^{\prime}=-\left(S^{2}-1+\delta^{2} / \alpha\right)^{1 / 2} \sin Y,
$$

where the negative square root is chosen so that $\theta^{\prime}$ is negative when $\theta$ decreases. A differentiation of (5.411) yields

$$
\theta^{\prime}=-\alpha^{-1}\left[\left(S^{2}-1\right) \alpha+\delta^{2}\right]^{1 / 2} Y^{\prime} \sin Y+\frac{1}{2}\left(d S^{2} / d \varphi\right)\left[\left(S^{2}-1\right) \alpha+\delta^{2}\right]^{-1 / 2} \cos Y .
$$

When the last two equations are equated, there is obtained

$$
Y^{\prime}=\alpha^{1 / 2}+\frac{1}{2} \alpha\left(d S^{2} / d \varphi\right)\left[\left(S^{2}-1\right) \alpha+\delta^{2}\right]^{-1} \cot Y .
$$

By (5.404) and (5.412) this last equation becomes

$$
Y^{\prime}(\varphi)=\alpha^{1 / 2}\left\{1-V_{2}^{\prime}(\theta) Z^{-1}\left[\left(S^{2}-1\right) \alpha+\delta^{2}\right]^{-1 / 2} \cos Y\right\},
$$

which is the third equation.

In this section the integration of (4.206) and (4.207) with the energy integral (4.102) has been reduced to the integration of the three first-order equations (5.404), (5.409) and (5.413). These three equations are essentially variations of the parameters of the solution found in Sec. 5.3. To check this, it is assumed that $\beta=\delta=0$, so that from (4.202) $V_{2}(\theta)=1$, and the three equations are immediately integrated as $S^{2}=$ constant, 
$X=S \varphi+$ constant, $Y=\alpha^{1 / 2} \varphi+$ constant; hence, (5.406) and (5.411) reduce to (5.302).

$5.5 A$ periodic solution. When $0<e<1$, the homogeneous solution (5.213) represents large-amplitude, periodic, anharmonic oscillations of an homogeneous star. In this section a similar solution for inhomogeneous stars is constructed which reduces to the homogeneous solution in the limit as $\delta$ approaches zero.

If the independent variable $x$ is defined as

$$
x=\sigma \varphi
$$

where $\sigma$ is a constant, then (4.206) and (4.207) become

$$
\begin{aligned}
\sigma^{2} Z^{\prime \prime}(x)+\left[1+\sigma^{2}\left(\theta^{\prime}(x)\right)^{2}+2 \delta \theta+\alpha \theta^{2}\right] Z & =1+\delta \theta+\frac{1}{2} \beta \theta^{2}, \\
\sigma^{2} Z \theta^{\prime \prime}(x)+(\alpha Z-\beta) \theta & =\delta(1-Z) .
\end{aligned}
$$

The constant $\sigma$ is considered to be an eigenvalue which is determined by the condition that the solution to (5.502) and (5.503) be periodic.

It is now assumed that $\delta$ is a small quantity whose cubes and higher powers may be neglected and the solution to (5.502) and (5.503) is expanded in powers of $\delta$ and truncated as

$$
\begin{aligned}
Z(x) & =Z_{0}(x)+\delta^{2} Z_{1}(x) . \\
\theta(x) & =\delta \theta_{0}(x), \\
\sigma^{2} & =1+\delta^{2} \sigma_{1} .
\end{aligned}
$$

There would be a considerable simplification if the quadratic terms in $\theta$ in (5.502) could be neglected. From (5.505) $\theta$ is assumed to be on the order of $\delta$; hence, all of the terms involving $\theta$ in (5.502) are of the same order, $\delta^{2}$. Therefore, the quadratic terms in (5.502) must be included to obtain the desired order of accuracy. The form of the expansions in (5.504), (5.505) and (5.506) is chosen so that this solution reduces to the homogeneous solution (5.213) as $\delta$ tends to zero.

When (5.504), (5.505) and (5.506) are substituted in (5.502) and (5.503) and corresponding powers of $\delta$ are equated, there is obtained in the order of increasing powers of $\delta$

$$
\begin{gathered}
Z_{0}{ }^{\prime \prime}+Z_{0}=1, \\
Z_{0} \theta_{0}{ }^{\prime \prime}+\left(\alpha Z_{0}-\beta\right) \theta_{0}=1-Z_{0}, \\
Z_{1}{ }^{\prime \prime}+Z_{1}=-\sigma_{1} Z_{0}{ }^{\prime \prime}-Z_{0}{\theta_{0}}{ }^{2}-\left(2 Z_{0}-1\right) \theta_{0}-\left(\alpha Z_{0}-\frac{1}{2} \beta\right) \theta_{0}{ }^{2} .
\end{gathered}
$$

Inclusion of higher powers of $\delta$ in (5.504) and (5.505) would yield equations whose whose operators on the left-hand side are of the same form as in (5.507) and (5.509) for the terms in $Z$ and as in (5.508) for the terms in $\theta$. The functions on the right-hand side of (5.508) and (5.509) are such that they depend only on quantities which are determined by the preceeding equations. This property would continue to hold if additional powers of $\delta$ were included in (5.504) and (5.505).

The solution to (5.507) is taken to be

$$
Z_{0}(x)=1+e \cos x
$$

where in this section $e$ is assumed to be the only non-zero constant of integration which lies in the range $0 \leq e<1$. 
Substitution of (5.510) in (5.508) gives

$$
(1+e \cos x) \theta_{0}{ }^{\prime \prime}+(\alpha-\beta+\alpha e \cos x) \theta_{0}=-e \cos x .
$$

Because (5.511) is a linear, second-order, inhomogeneous differential equation, the general solution to (5.511) is the sum of the solutions to its homogeneous and inhomogeneous parts. The homogeneous part of (5.511) is Hill's differential equation (Hill [9], McLachlan [10]). Methods of solution and determining the stability of Hill's equation are known and are not pursued here. Whether the solution to the homogeneous part of (5.511) is stable or unstable determines whether solutions to (5.511) "near" the periodic solution constructed next are stable or unstable. In addition, the general solution to the homogeneous part of (5.511) is suppressed because it would involve two additional constants of integration whereas $e$ is assumed to be the only non-trivial constant of integration in this section.

Since (5.511) only involves $\cos x$, the solution to the inhomogeneous part of (5.511) is assumed to be a Fourier cosine series, namely

$$
\theta_{0}(x)=\sum_{n=0}^{\infty} a_{n} \cos n x
$$

Substitution of (5.512) in (5.511) yields the following recursion relations for the $a_{n}$ s when the separate coefficients of $\cos n x$ are equated:

$$
\begin{aligned}
2(\alpha-\beta) a_{0}+(\alpha-1) e a_{1} & =0 \\
\alpha e a_{0}+(\alpha-\beta-1) a_{1}+\frac{1}{2}(\alpha-4) e a_{2} & =-e \\
{\left[(n-1)^{2}-\alpha\right] e a_{n-1}+2\left(n^{2}-\alpha+\beta\right) a_{n}+\left[(n+1)^{2}-\alpha\right] e a_{n+1} } & =0, \quad n \geq 2 .
\end{aligned}
$$

When (5.510) is substituted in (5.509) there comes

$Z_{1}{ }^{\prime \prime}+Z_{1}=\sigma_{1} e \cos x-(1+. e \cos x) \theta_{0}{ }^{2}-(1+2 e \cos x) \theta_{0}-\left(\alpha-\frac{1}{2} \beta+\alpha e \cos x\right) \theta_{0}{ }^{2}$.

To solve this differential equation it is necessary to square (5.512) and its derivative; hence,

$$
\theta_{0}{ }^{2}=\sum_{n=0}^{\infty} d_{n} \cos n x, \quad{\theta_{0}}^{2}=\sum_{n=0}^{\infty} f_{n} \cos n x
$$

where

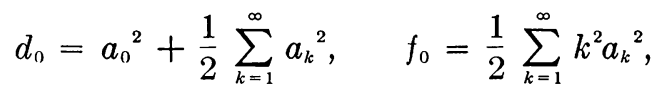

$$
\begin{aligned}
& d_{n}=\frac{1}{2}\left[a_{0} a_{n}+\sum_{k=0}^{\infty}\left(a_{|n-k|}+a_{n+k}\right) a_{k}\right], \quad n \geq 1, \\
& f_{n}=\frac{1}{2} \sum_{k=1}^{\infty}\left[(k-n) a_{|k-n|}+(k+n) a_{k+n}\right] k a_{k}, \quad n \geq 1 \text {. }
\end{aligned}
$$

The solution to (5.514) is assumed to be the Fourier cosine series

$$
Z_{1}(x)=b_{0}+\sum_{n=2}^{\infty} b_{n} \cos n x .
$$

A term in $\cos x$ is not included in (5.517) because its coefficient would be an arbitrary 
constant whereas $e$ in (5.510) is assumed to be the only constant of integration. Substitution of (5.512), (5.515) and (5.517) in (5.514) yields, when the separate coefficients of $\cos n x$ are equated,

$$
\begin{aligned}
& b_{0}=-\left[a_{0}+\left(\alpha-\frac{1}{2} \beta\right) d_{0}+f_{0}+\frac{1}{2} e\left(2 a_{1}+\alpha d_{1}+f_{1}\right)\right], \\
& \sigma_{1}=2 a_{0}+a_{2}+\alpha\left(d_{0}+\frac{1}{2} d_{2}\right)+f_{0}+\frac{1}{2} f_{2}+\left[a_{1}+\left(\alpha-\frac{1}{2} \beta\right) d_{1}+f_{1}\right] / e, \\
& b_{n}=\left\{a_{n}+\left(\alpha-\frac{1}{2} \beta\right) d_{n}+f_{n}+\frac{1}{2} e\left[2\left(a_{n-1}+a_{n+1}\right)\right.\right. \\
& \left.\left.\quad+\alpha\left(d_{n-1}+d_{n+1}\right)+f_{n-1}+f_{n+1}\right]\right\} /\left(n^{2}-1\right), \quad n \geq 2,
\end{aligned}
$$

The Fourier coefficients $a_{n}$ and $b_{n}$ which result when (5.513), (5.518) and (5.520) are computed to order $e^{3}$ are listed in Table 1 along with the eigenvalue $\sigma_{1}$ from (5.519). A periodic solution to (4.206) and (4.207), correct to order $e$, is found from Table 1, (5.517), (5.512), (5.510), (5.504) through (5.506) and (5.501) to be

$$
\begin{aligned}
Z & =1+e \cos \left\{\left[1-\delta^{2} / 2(\alpha-\beta-1)\right] \varphi\right\}, \\
\theta & =-[\delta e /(\alpha-\beta-1)] \cos \left\{\left[1-\delta^{2} / 2(\alpha-\beta-1)\right] \varphi\right\} .
\end{aligned}
$$

This solution may be directly verified by substitution in (4.206) and (4.207), and its corresponds to one of the normal mode solutions which result when (4.206) and (4.207) are linearized.

TABLE 1. Fourier coefficients correct to order $e^{3}$.

$$
\begin{aligned}
& a_{0}=\frac{(\alpha-1) e^{2}}{2(\alpha-\beta)(\alpha-\beta-1)} \\
& a_{1}=-\frac{e}{\alpha-\beta-1}\left[1+\frac{(\alpha-1) e^{2}}{2(\alpha-\beta-1)}\left(\frac{\alpha-\beta}{\alpha-\beta}+\frac{\alpha-4}{2(\alpha-\beta-4)}\right)\right] \\
& a_{2}=\frac{(\alpha-1) e^{2}}{2(\alpha-\beta-1)(\alpha-\beta-4)} \\
& a_{3}=\frac{(\alpha-1)(\alpha-4) e^{3}}{4(\alpha-\beta-1)(\alpha-\beta-4)(9-\alpha+\beta)} \\
& d_{0}=f_{0}=d_{2}=-f_{2}=\frac{e^{2}}{2(\alpha-\beta-1)^{2}} \\
& d_{1}=-\frac{(\alpha-1) e^{3}}{(\alpha-\beta)(\alpha-\beta-1)^{2}+2 f_{1}} \\
& f_{1}=2 d_{3}=-f_{3}=-\frac{(\alpha-1) e^{3}}{(\alpha-\beta-1)^{2}(\alpha-\beta-4)} \\
& b_{0}=-\frac{e^{2}}{2(\alpha-\beta-1)}\left[\frac{\alpha-1}{\alpha-\beta}+\frac{\alpha-\frac{1}{2} \beta+1}{\alpha-\beta-1}-2\right] \\
& b_{2}=\frac{e^{2}}{6(\alpha-\beta-1)}\left[\frac{\alpha-\frac{1}{2} \beta-1}{\alpha-\beta-1}+\frac{\alpha-1}{\alpha-\beta-4}-2\right] \\
& b_{3}=-\frac{(\alpha-1) e^{3}}{32(\alpha-\beta-1)(\alpha-\beta-4)}\left[\frac{\alpha}{\alpha-\beta-1}-\frac{\alpha-4}{9-\alpha+\beta}-2\right] \\
& \sigma_{1}=-\frac{1}{\alpha-\beta-1}\left[1+\frac{(\alpha-1) e^{2}}{\alpha-\beta-1}\left(\frac{\alpha+\beta+2}{2(\alpha-\beta)}-\frac{3 \alpha+1}{\alpha-1}+\frac{9 \alpha-4 \beta-2}{4(\alpha-\beta-4)}\right)\right]
\end{aligned}
$$




\section{A numerical example.}

6.1 An inhomogeneous star. To illustrate the preceeding theory, consider the simple inhomogeneous density distribution

$$
\rho_{0}(\xi)=15 M\left(1-\xi^{2}\right) / 8 \pi,
$$

where $M$ is the total mass of the star. Following the summary given at the end of Sec. 3.5, the mass and pressure distributions are found from (2.404) and (2.405) to be

$$
m(\xi)=\frac{1}{2} M \xi^{3}\left(5-3 \xi^{2}\right), \quad P_{0}(\xi)=15 G M^{2} R_{0}\left(1-\xi^{2}\right)^{2}\left(2-\xi^{2}\right) / 32 \pi .
$$

From (3.202), (3.302) and (3.402) there come

$$
\rho_{c}=3 M / 28 \pi, \quad P_{c}=5 G M^{2} R_{0} / 84 \pi, \quad K=5 / 3 .
$$

The functions and the values of the constants obtained in this section should be compared with the results for a homogeneous star found in Sec. 5.2.

The function $u(a)$ can be evaluated in terms of elementary functions from the definition (3.202) and (6.101) as

$$
u(a)=\frac{35}{2}\left[-1 / 5 a-(1-a) 3 a^{2}+(1-a)\left(1-u_{0}(a)\right) / a^{3}\right],
$$

where $u_{0}(a)$ is given by (5.205). The formula (6.104) is not useful for numerical evaluation near $a=0$. A power series for $u(a)$ which converges for $|a|<1$ is derived from (3.202) as

$$
u(a)=35 \sum_{n=0}^{\infty}(-1)^{n} a^{n} /(2 n+5)(2 n+7) .
$$

From this series the values of $u^{\prime}(0), u^{\prime \prime}(0)$ and $u^{\prime \prime \prime}(0)$ are easily obtained. From (5.207) and $(6.105)$ the relation between $a$ and $\theta$ correct to order $\theta^{2}$ is obtained as

$$
a(\theta)=9.43927 \theta+47.2152 \theta^{2} .
$$

The information needed about the potentials $V_{1}(\theta)$ and $V_{2}(\theta)$ is contained in the constants $\alpha, \beta$, and $\delta$. From (4.203), (4.204) and (4.205) there is obtained

$$
\alpha=12.1727, \quad \beta=4.56503, \quad \delta=-0.190692 \text {, }
$$

which should be compared with (5.209). For the constants (6.107), the equilibrium solutions (5.102) and (5.101) are

$$
\begin{array}{r}
Z=1, \quad \theta=0 ; \quad Z=0.356556, \quad \theta=0.546051 ; \\
Z=0.394043, \quad \theta=-0.499047,
\end{array}
$$

as compared with the values for a homogeneous star given by (5.210).

6.2 Curves of constant energy. One constant of integration for Eqs. (4.103) and (4.104) is known from the total energy of the star (4.102). This integral determines regions in the $(Z, \theta)$ plane in which the solution sets of (4.103) and (4.104) must lie. For any given value of $E$, any point $(Z, \theta)$ may or may not be a member of the solution set according to whether the kinetic energy $\frac{1}{2}\left(Z^{\prime 2}+Z^{2} \theta^{\prime 2}\right)$ as computed from (4.102) is positive or negative. If the kinetic energy is positive, the point $(Z, \theta)$ can be a point of the solution for the given value of $E$. If the kinetic energy is negative, the point $(Z, \theta)$ cannot be a point in the solution. The energy integral is called an "isolating integral" because it isolates regions in the $(Z, \theta)$ plane which may or may not be points in a solution set for a given value of $E$. These regions are determined from (4.102) by setting the 
kinetic energy equal to zero; namely

$$
\frac{1}{2} V_{1}(\theta) Z^{2}-V_{2}(\theta) Z=E .
$$

This quadratic equation is solved for $Z$ as

$$
Z_{E}(\theta)=\left\{V_{2}(\theta) \pm\left[V_{2}^{2}(\theta)+2 E V_{1}(\theta)\right]^{1 / 2}\right\} / V_{1}(\theta) .
$$

For a given $E$, (6.201) determines a locus of points in the $(Z, \theta)$ plane along which the kinetic energy is zero.

In Fig. 1, the curves (6.201) are plotted for the density (6.101). The potentials $V_{1}(\theta)$ and $V_{2}(\theta)$ are computed from (4.201) and (4.202) by use of the constants (6.107). The point $Z=1, \theta=0$ is obtained from (6.201) for the energy $E=-0.5$. The curves are constructed for values of $E$ in the range [ $-0.5,0]$ in increments of 0.05 . The equilibrium points (6.108) are indicated in Fig. 1 by crosses.

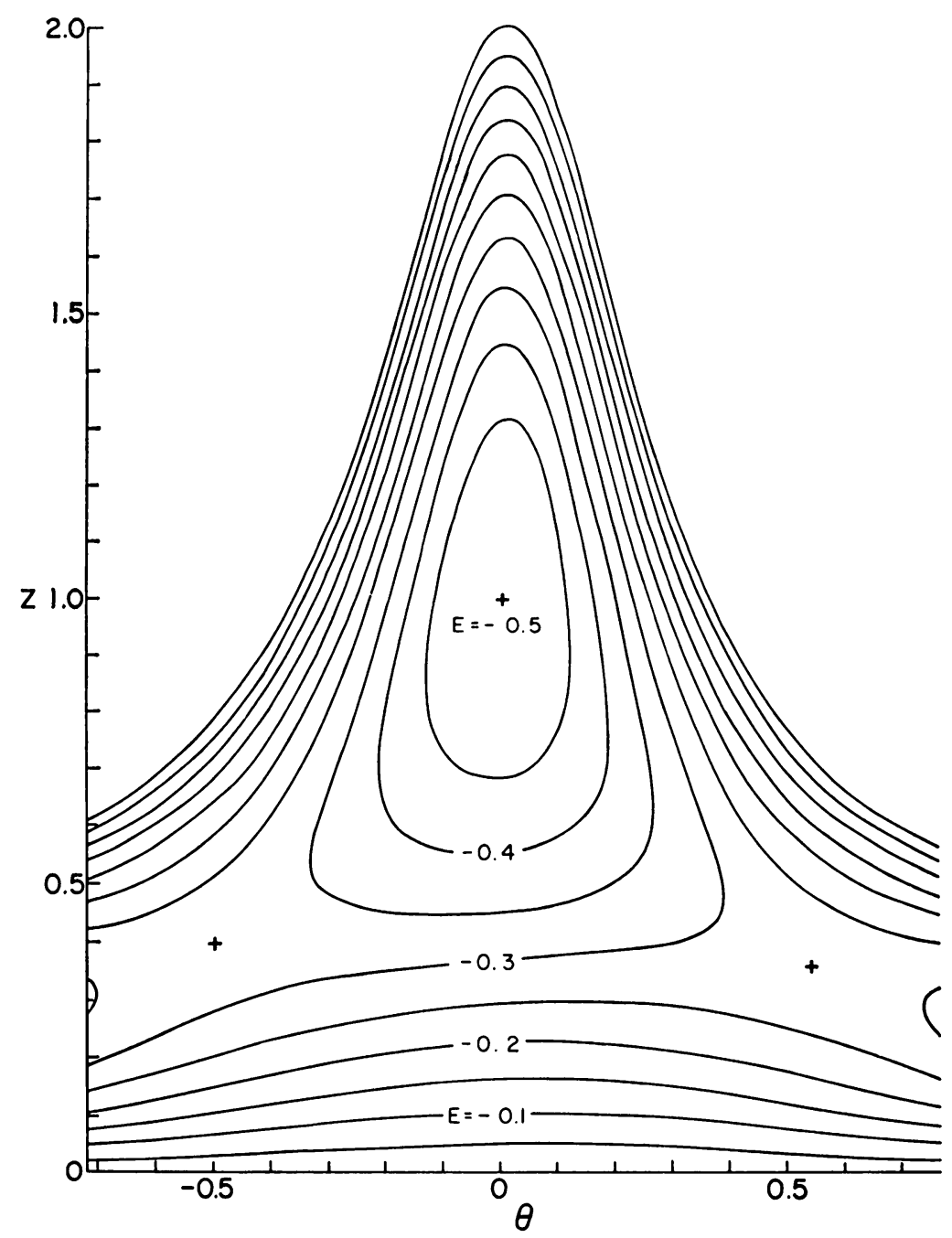

FIG. 1. Constant energy curves. 
An equilibrium point is a point of stable equilibrium if curves of constant energy enclose the equilibrium point. An equilibrium point is a point of unstable equilibrium if the curves do not enclose the point. From an examination of Fig. 1, it is apparent that $Z=1, \theta=0$ is a point of stable equilibrium whereas the other two points are points of unstable equilibrium. For example, if initially $Z=1, \theta=0$ and $E=-0.45$, then for all values of $\varphi$ the solution set $(Z(\varphi), \theta(\varphi))$ must lie within the curves marked by $E=$ -0.45 ; hence, all values of $Z$ and $\theta$ must remain "near" $Z=1, \theta=0$. On the other hand, if initially $Z=0.356, \theta=0.546$ and $E=-0.25$, then the solution set $(Z(\varphi), \theta(\varphi))$ must remain inside of the curve denoted by $E=-0.25$; hence, all values of $Z$ and $\theta$ are not necessarily "near" the original values.

6.3 Numerical solutions of first-order equations. In Fig. 2 a solution set of (4.206) and (4.207) is plotted. This solution was obtained by numerical integration of (5.404),

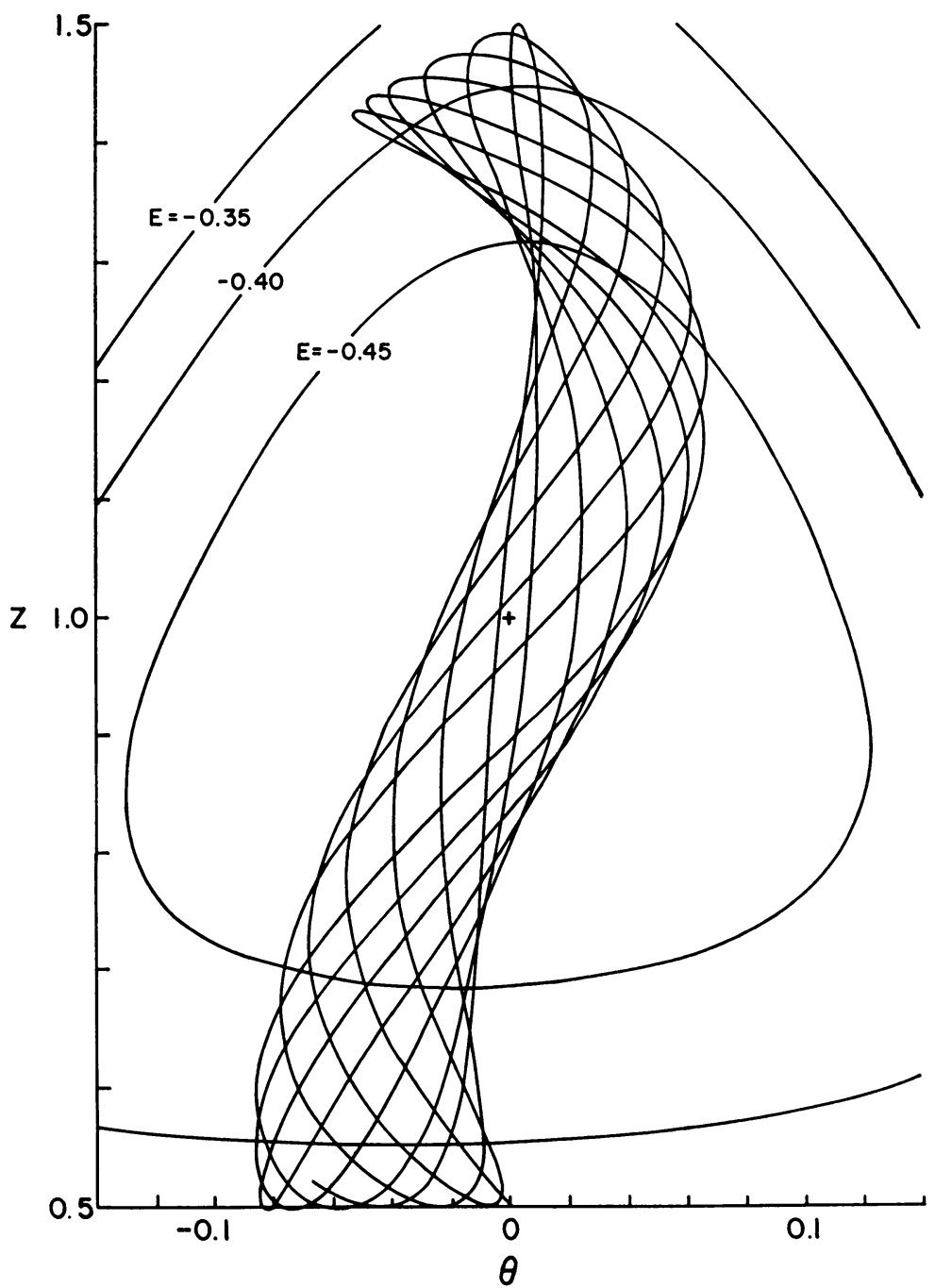

Fig. 2. The orbit of a solution to the quasi-linear equations. 
(5.409) and (5.413). This integration yields $S(\varphi), X(\varphi)$ and $Y(\varphi)$ which upon substitition in (5.406) and (5.411) yield $Z(\varphi), \theta(\varphi)$. The initial values are $Z=0.5, \theta=Z^{\prime}=\theta^{\prime}=0$; hence, from (6.201), $E=-0.375$. Since the scales of Figs. 1 and 2 are different, the constant-energy curves for $E=-0.45,-0.40$ and -0.35 are included in Fig. 2 for reference.

Unless something pathological occurs, it appears from Fig. 2 that this solution set is contained within some kind of a curvilinear "box". That is, there is a box-like region of the $Z, \theta$ plane in which this solution set lies. Since this box does not include all values of $Z$ and $\theta$ which are within the constant-energy curve for $E=-0.375$, there is apparently some other isolating integral which bounds $Z$ and $\theta$ within a region smaller than that allowed by the energy integral.

A similar situation occurs for the approximate solution of Sec. 5.3. When $\beta=\delta=0$, $E$ and $S$ of (5.301) are constants of integration which provide isolating integrals. Any solution to (4.206) and (4.207) in this case must lie within the region of the $Z, \theta$ plane enclosed by the appropriate constant energy curve computed from (6.201). But in addition this solution must satisfy both equations of (5.301); hence, from (5.302) the values of $Z$ and $\theta$ must satisfy $\left[1-\left(1+2 S^{2} E\right)^{1 / 2}\right] / S^{2} \leq Z \leq\left[1+\left(1+2 S^{2} E\right)^{1 / 2}\right] / S^{2}$ and $-\left[\left(S^{2}-1\right) / \alpha\right]^{1 / 2} \leq \theta \leq\left[\left(S^{2}-1\right) / \alpha\right]^{1 / 2}$. Thus, in this simple case the second isolating integral requires the solution set to lie within a rectangular box in the $(Z, \theta)$ plane.

It is not known how a second isolating integral for the more general case when $\beta$ and $\delta$ are nonzero may be constructed, or even if such a construction is possible in general. If it is possible, the construction will be complicated by the existence of the two additional points of unstable equilibrium which are present in the more general case. The boundary of the smaller isolating region must be some kind of a singular solution of the energy integral (4.102) considered as a non-linear differential equation.

Upon examination of the orbit plotted in Fig. 2, it occurred to the author that it might be posslble to obtain a periodic solution if one could but "squeeze" together the curved sides of the box. To test this hypothesis, another numerical integration was performed with the initial conditions $Z=0.497, \theta=-0.045, Z^{\prime}=\theta^{\prime}=0$. Again the orbit which resulted appeared to lie within a curvilinear box. This box is plotted in Fig. 3 . Although a single curve was not produced, the size of the box in Fig. 3 is smaller than that of Fig. 2. This result suggested the theory of Sec. 5.5.

6.4 Periodic solutions. The theory of Sec. 5.5 was tested numerically for various values of the skewness parameter $e$. Four of the solutions $Z(\varphi), \theta(\varphi)$ which were obtained for $e=0.2,0.3,0.4$, and 0.5 are plotted in Fig. 4. These solutions were constructed by solving (5.513) as a set of linear algebraic equations for the first twenty $a_{n}$ 's. Since these are linear equations, Krammer's rule was used to solve them. The determinant of the coefficients of the first twenty $a_{n}$ s is given below in Table 2 under the heading $\Delta$. The

TABLE 2.

\begin{tabular}{clllr}
\hline$e$ & \multicolumn{1}{c}{$E$} & \multicolumn{1}{c}{$\sigma$} & \multicolumn{1}{c}{$\Delta$} & \multicolumn{1}{c}{$I / \sigma$} \\
\hline 0 & -0.5 & 1.0 & 1.0 & 6.2832 \\
0.1 & -0.4950 & 0.9972 & 0.9608 & 6.4296 \\
0.2 & -0.4801 & 0.9968 & 0.8482 & 6.7720 \\
0.3 & -0.4551 & 0.9964 & 0.6780 & 7.3891 \\
0.4 & -0.4200 & 0.9963 & 0.4763 & 8.4252 \\
0.5 & -0.3789 & 0.9992 & 0.2767 & 10.3168
\end{tabular}




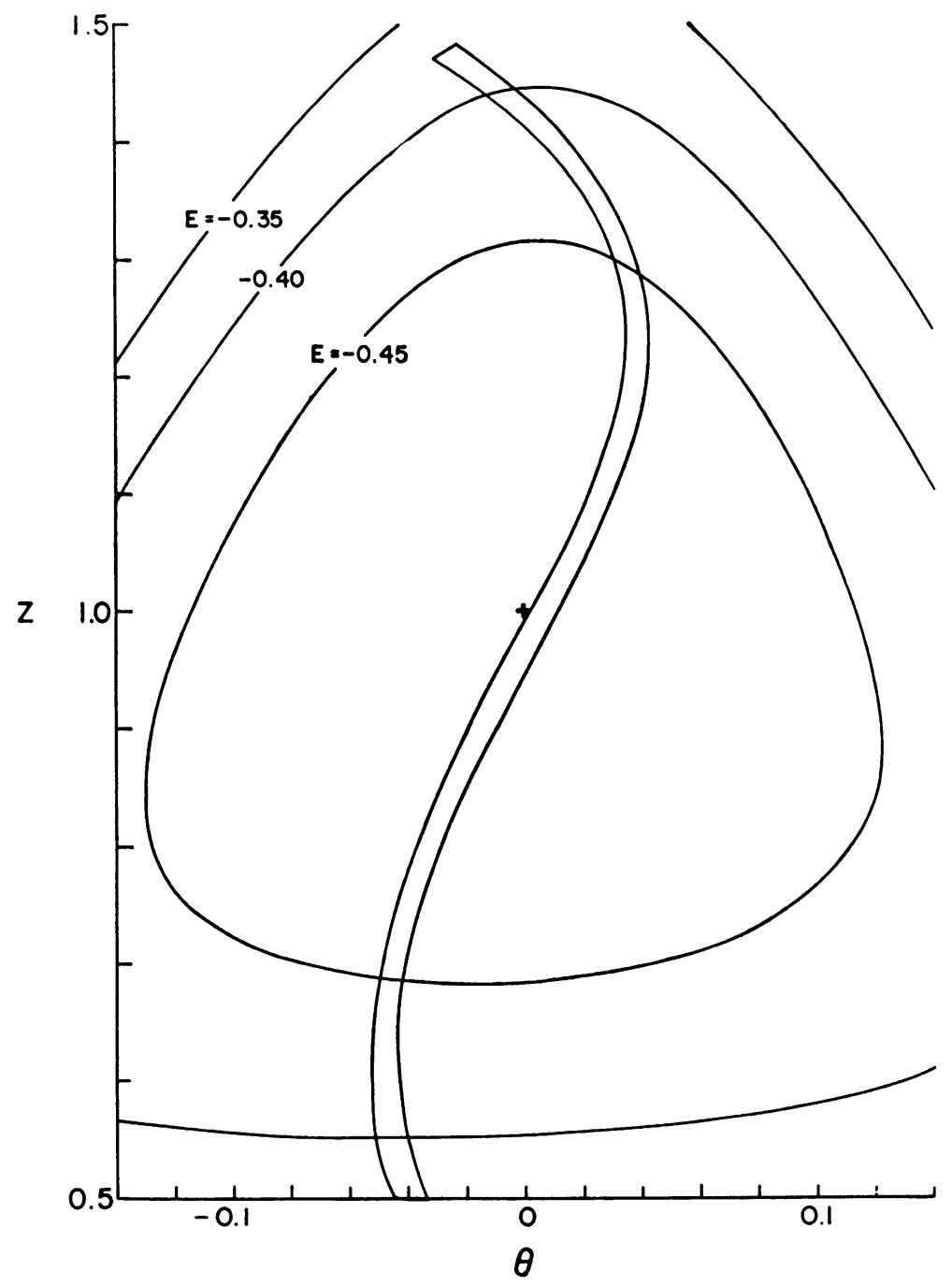

Fig. 3. The curvilinear box containing another solution to the quasi-linear eqautions.

$a_{n} \mathrm{~s}$ were then used to compute the first twenty $b_{n} \mathrm{~s}$ from (5.518) and (5.520). The values of $\sigma_{1}$ were computed from (5.519), $\sigma$ was then computed from (5.506), and its values are given in Table 2. With the $a_{n} \mathrm{~s}$ and $b_{n}$ s computed $Z(x)$ and $\theta(x)$ were computed next using (5.517), (5.512), (5.510), (5.504) and (5.505).

The orbit for $e=0.5$ in Fig. 4 should be compared with the orbit in Fig. 2 and the curvilinear box in Fig. 3. Since this curve seems to result when the sides of the curvilinear boxes of Figs. 2 and 3 are "squeezed" together, the periodic solution of Sec. 5.5 for $e=$ 0.5 is stable.

It was not possible to construct these periodic solutions for larger values of $e$ as the approximations of Sec. 5.5 began to break down. It is apparent from Table 2 that $\Delta$ decreases with increasing $e$, and in fact a root was determined graphically to occur at 


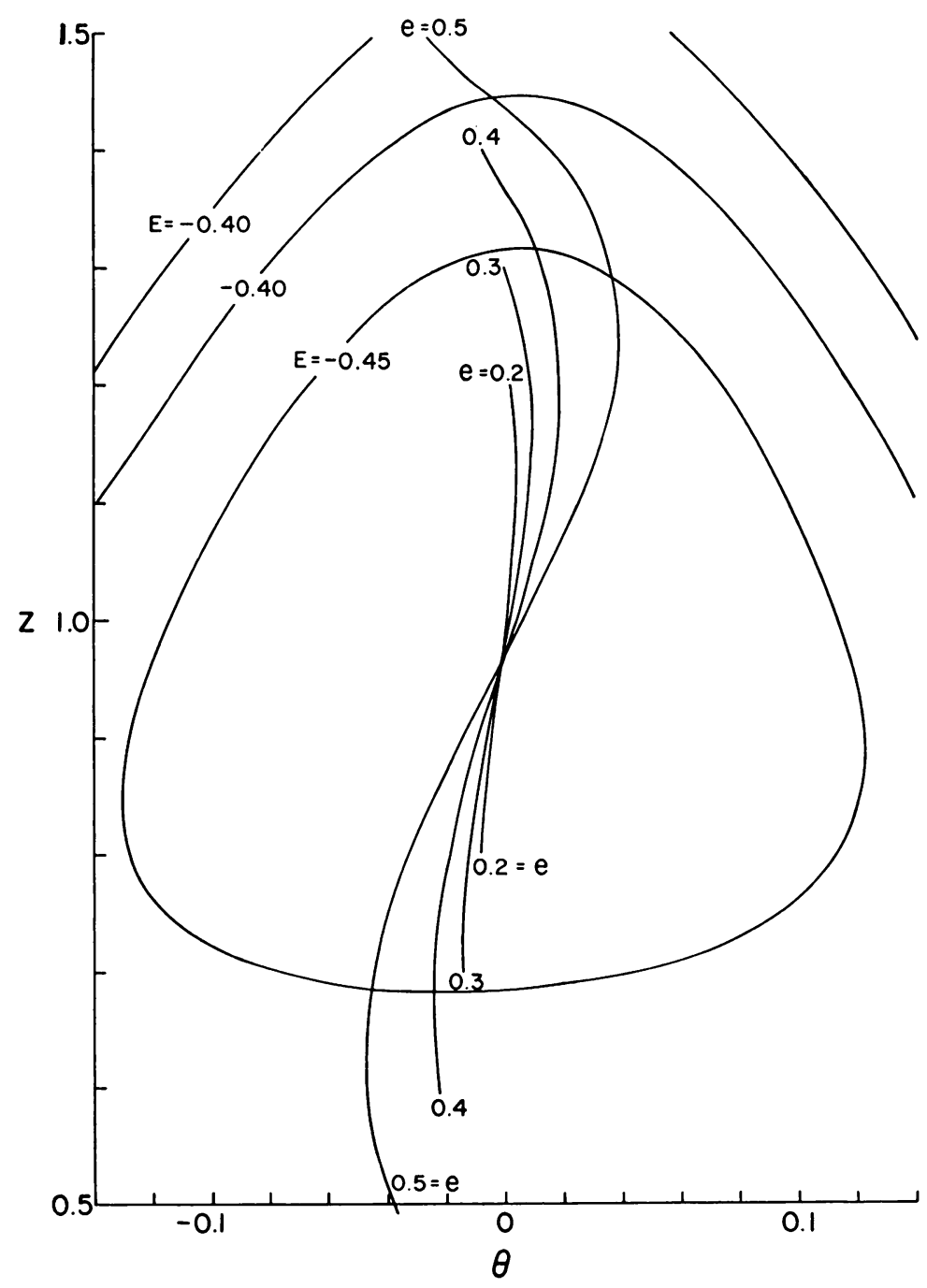

FIG. 4. The orbits of four periodic solutions to the quasi-linear equations.

$e \simeq 0.71$. The Fourier series (5.512) does not provide a solution to (5.511) at the value of $e$ for which $\Delta=0$. The accuracy of the solutions was checked by computing the energy $E$ from (4.102) for each value of $x$, and for values of $e$ larger than 0.5 , the energy ceased to be reasonably constant. For accurate solutions for values of $e$ in the range $0.5<e<0.7$ more terms must be included in the expansions (5.504), (5.505) and (5.506).

6.5 Observable quantities. In order to compare the theory of this paper with observations of variable stars, it is necessary to obtain quantities that are directly observable. Since radiative processes are neglected, the only remaining observables are the variable star's period, radial variation and velocity variations. The star's radius as a function of time is obtained from (2.203), (3.203) and (4.101) as

$$
r_{b}(t) / R_{0}=Z^{-1}[u(a)(1+a)]^{-1 / 2} .
$$


If the right-hand side of the last equation is expanded to order $\theta^{2}$, there is obtained

$$
r_{b}(t) / R_{0}=\left(1+C_{1} \theta+\frac{1}{2} C_{2} \theta^{2}\right) / Z,
$$

where the constants $C_{1}$ and $C_{2}$ are computed from

$$
\begin{aligned}
& C_{1}=-[2 / w(0)]^{1 / 2}\left[1+u^{\prime}(0)\right], \\
& C_{2}=2\left\{\left[w^{\prime}(0) / w(0)+u^{\prime}(0)+3\right]\left[1+u^{\prime}(0)\right]-2\left[2 u^{\prime}(0)+u^{\prime \prime}(0)\right]\right\} / w(0) .
\end{aligned}
$$

For the density (6.101) these constants are

$$
C_{1}=-2.09762, \quad C_{2}=26.7538 \text {. }
$$

A time derivative of (6.501) yields the velocity of the boundary as

$$
\dot{r}_{b}(t) T_{0} / R_{0}=-Z^{\prime}(\varphi)\left(1+C_{1} \theta+\frac{1}{2} C_{2} \theta^{2}\right)+Z\left(C_{1}+C_{2} \theta\right) \theta^{\prime}(\varphi),
$$

where the chain rule and the second transformation of (4.101) have been used.

To obtain the time dependence of (6.501) and (6.502), it is necessary to integrate the second equation of (4.101) to obtain $t(\varphi)$. In general this integration must be carried out numerically, although it can be done exactly in some approximations (see Sec. 5.2). Eq. (4.101) can be rewritten as

$$
\left(t-t_{0}\right) / T_{0}=\int_{0}^{\varphi} Z^{-2}(\varphi) d \varphi,
$$

where $t_{0}$ is a constant. When the solution for $Z(\varphi), \theta(\varphi)$ is periodic in $\varphi$, the solution is periodic in $t$. For the periodic solution of Sec. 5.5 , the last integral yields the time period $T$ as

$$
T=T_{0} I / \sigma, \quad I=\int_{0}^{2 \pi} Z^{-2}(x) d x,
$$

where (5.501) has been used and $Z(x)$ is given by (5.504). It is customary in variable star observations to present data in terms of fractions of the star's period. Thus, the "phase"

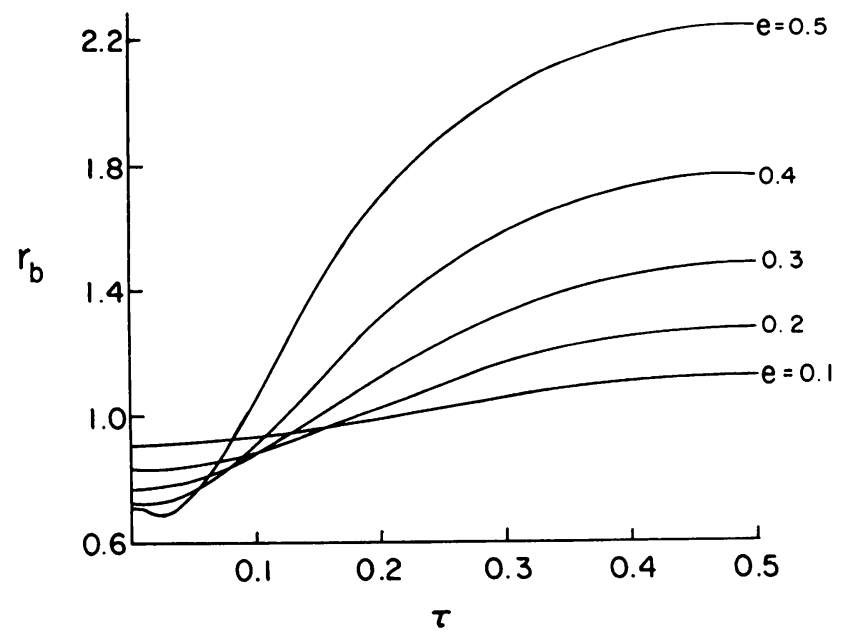

FIG. 5. The radius of the star for the periodic solutions. 
$\tau$ is defined as

$$
\tau=\left(t-t_{0}\right) / T=I^{-1} \int_{0}^{x} Z^{-2}(x) d x .
$$

From (4.10j) and (6.j03) there comes

$$
T=(I / \sigma) R_{0}^{3 / 2}(G M K)^{-1 / 2},
$$

which relates the period to the mass, radius and structure of the star and to the amplitude of its oscillation. This formula should be compared with the results for a homogeneous star (5.214).

Values of $I / \sigma$ are given in Table 2 for the computations described in Sec. 6.4. In Figs. 5 and 6 , the radius of the star $r_{b}$ defined by (6.501) and its velocity $\dot{r}_{b}$ defined by (6.502) are plotted as functions of $\tau$ given by (6.504) for the periodic solutions of Sec. 6.4. Only the variations over a half-period are graphed because the radius is an even function of $\tau$ whereas the radial velocity is an odd function of $\tau$, and, therefore, the variation over a full period is readily deduced. The unit of radius in Fig. 5 is $R_{0}$, and the unit of velocity in Fig. 6 is $R_{0} / T$.

In Melvin [11] it is shown that the homogeneous solution (5.213) fits the observed adiabatic velocity curve of $\delta$ Cephei to within a $5 \%$ error. In a later investigation it will be attempted to reduce further the residuals of this fit by use of the radial velocity curve (6.502).

Besides [11] there have been several attempts to devise a "simple" analytical explanation for Cepheid variations. One of the more successful is the model of Rudd and Rosenberg [12]. The models of [11] and [12] are derived under the assumption that the nonadiabatic limit cycle is governed by a single, nonlinear oscillator equation. The reduction to a single oscillator is, however, equivalent to the assumption that there is a standing wave solution to the hydrodynamical equations for a pulsating star. The investigation of this paper shows that such a result is unlikely because it neglects the coupling between the modes which is caused by the inhomogeneity of the star.

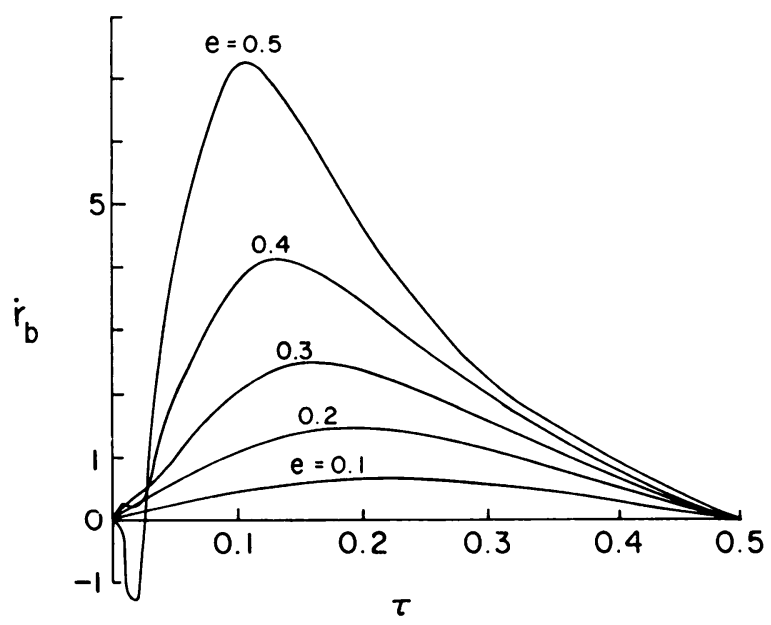

FIg. 6. Radial velocity curves for the periodic solutions. 
It appears likely from (4.203) that the coupling constant $\delta$ can vanish only for a homogeneous star. This means that a periodic solution must be a travelling wave which is governed by several coupled nonlinear oscillator equations. It is not obvious that such equations even admit large-amplitude periodic solutions. The work of Rosenberg [13] shows that nonlinear normal mode solutions can be constructed for certain two-dimensional potentials. The investigation of this paper shows that a fundamental, nonlinear normal-mode solution can be constructed for the equations of a star (3.502) and (3.503) by use of the theory of Sec. 5.5.

When the velocity curves of Fig. 6 are compared with those for a homogeneous star (see Rosseland [1], or Cox [14]), it is seen that the effect of the inhomogeneity can be quite large. The largest differences occur near the phase of minimum radius when the material of the star is the most compressed and the secondary wave travels the most rapidly.

In this age of the large digital computer it may seem to be an anachronism to develop an analytical theory of pulsating stars. Cox [14] notes, however, that the "brute-force" methods of numerical integration have not been entirely successful, especially without an analytical solution with which the numerical results may be compared. It is not necessary to use a large digital computer to construct the periodic solutions of Sec. 5.5; although it is tedious, a pocket calculator is sufficient.

\section{REFERENCES}

[1] S. Rosseland, The pulsation theory of variable stars, Dover, New York, 1964, pp. 99-102

[2] G. C. McVittie, Astron. J. 61, 451 (1956)

[3] M. J. Disney, D. McNally and A. E. Wright, Mon. Not. R. Astr. Soc. 140, 319 (1968)

[4] C. C. Lin, L. Mestel and F. H. Shu, Astrophys. J. 142, 1431 (1965)

[5] M. Fujimoto, Astrophys. J. 152, 523 (1968)

[6] J. P. Cox and R. T. Giuli, Principles of stellar structure, Vol. II, Gordon and Breach, New York, 1968 , p. 644 ff.

[7] H. Goldstein, Classical mechanics, Addison and Wesley, Reading, 1950, p. 217

[8] J. M. A. Danby, Celestial mechanics, Macmillan, New York, 1963, p. 56 ff.

[9] G. W. Hill, Acta Math. 8, 1 (1886)

[10] N. W. McLachlan, Theory and application of Mathieu functions, Dover, New York, 1964, p. 127 ff.

[11] P. J. Melvin, A homogeneous, nonadiabatic model of Delta Cephei, to appear in An international symposium on dynamical systems, ed. L. Cesari and A. R. Bednarek, Academic Press, New York, 1977

[12] T. J. Rudd and R. M. Rosenberg, Astron. and Astrophys. 6, 193 (1970)

[13] R. M. Rosenberg, Quart. Appl. Math. 22, 217 (1964)

[14] J. P. Cox, Rep. Prog. Phys. 37, 563 (1974) 